Article

\title{
Quantitative Studies on PDMS-PDMS Interface Bonding with Piranha Solution and its Swelling Effect
}

\author{
Kai-Seng Koh, Jitkai Chin *, Joanna Chia and Choon-Lai Chiang \\ Department of Chemical and Environmental Engineering, University of Nottingham Malaysia Campus, \\ Jalan Broga, Semenyih 43500, Selangor, Malaysia; E-Mails: koh.kaiseng@nottingham.edu.my (K.-S.K.); \\ joanna.chia@nottingham.edu.my (J.C.); cl.chiang@nottingham.edu.my (C.L.C.) \\ * Author to whom correspondence should be addressed; E-Mail: jit-kai.chin@nottingham.edu.my; \\ Tel.: +6-038-924-8378; Fax: +6-038-924-8017.
}

Received: 10 March 2012; in revised form: 26 April 2012 / Accepted: 26 April 2012 /

Published: 4 May 2012

\begin{abstract}
In this paper, a low-cost yet effective method of irreversible bonding between two elastomeric polydimethylsiloxane (PDMS) interfaces using Piranha solution is investigated. Piranha solutions at a weight ratio of 3:1 using different acids and hydrogen peroxide were attempted. The average tensile strengths of the device bonded with concentrated sulfuric acid-based piranha solution and nitric acid-based piranha solution were found to be $200 \pm 20 \mathrm{kPa}$ and $100 \pm 15 \mathrm{kPa}$ respectively. A PDMS surface treated with Piranha Solution demonstrated an increase in hydrophilicity. In addition, relatively straightforward swelling studies of PDMS using a weight loss method with common organic solvents were also investigated. Experimental results show that hexane, toluene, ethyl acetate, n-propyl alcohol and acetone swell PDMS significantly over a duration of up to $1 \mathrm{~h}$ and above; PDMS samples reached a steady state of swelling only after $5 \mathrm{~min}$ of immersion in other solvents. This will enable researchers to develop devices for the future according to the interaction between the material and the solvents in contact.
\end{abstract}

Keywords: polydimethylsiloxane (PDMS); swelling; bonding effects; piranha solution 


\section{Introduction}

Utilisation of Polydimethyl Siloxane (PDMS) as raw material for microfluidic fabrication has been extensively reported in various fields [1-4]. However, microfabrication of the devices, especially using soft-lithography methods remains a challenge for researchers, particularly those with the necessary sophisticated configuration. Although various high-end equipments are frequently used to ensure high quality of fabrication products, they are expensive and unaffordable for many researchers with limited resources, especially those from developing countries.

PDMS-PDMS interface bonding techniques such as hydrophobic bonding, oxygen plasma $[5,6]$, corona discharge [7], partial curing method etc., have been used commonly and are still being optimized in the fabrication of PDMS-based microfluidic devices. Uncured PDMS adhesive is the method that provides maximum bonding strength of approximately $671 \mathrm{kPa}$, followed by partial curing and then the oxygen plasma method. Bonding strength by the Corona Discharge method is $290 \mathrm{kPa}$ [8]. UV exposure is also one of the low cost solutions and widely used in a laboratory without clean room facilities. Bondings based on these methods are robust and near permanent. Also, these methods are not only highly appropriate for PDMS-PDMS homogenous bonding, but also possess broad applicability for numerous substrates such as glass, metal and silicon wafers [9].

There are many drawbacks associated with these methods, including the requirements of a low-vacuum environment in the presence of oxygen gas for exposed bonding surfaces and clean room facilities for the oxygen plasma technique. Furthermore, reliability and repeatability of these techniques is a controversial theme as there is a fluctuation up to $50 \%$ in the bonding strength using oxygen plasma [5] and 20\% in utilizing the corona discharge method respectively [5].

PDMS layers can be adhered both reversibly and irreversibly, depending on the technique used. Reversible bonding methods are only suitable for low pressure applications, because the Van der Waals bonding is too weak, which is not suitable for applications higher than $35 \mathrm{kPa}$ [10]. At the same time, an irreversible bonding technique involves material surface modifications, and is generally able to sustain relatively higher pressures, typically $100 \mathrm{kPa}$ and above. It is generally compatible with most chemicals and biological applications, hence the irreversible bonding method has become the main research focus.

One of the low cost but rarely reported methods is utilising piranha solution as the bonding reagent. Piranha solution is a strong oxidizer normally consisting of concentrated sulfuric acid and hydrogen peroxide, commonly known as a cleaning agent in wafer cleaning in the soft-lithography process. The Piranha solution is also known for changing the surface properties of polymers, such as PDMS, comprised of repeated units of $-\mathrm{O}-\mathrm{Si}\left(\mathrm{CH}_{3}\right)_{2}-$, in which silanol groups are developed when the surface is oxidised. The silanol groups are highly polar, resulting in a surface layer which is highly hydrophilic. When two such layers are brought into contact, the $\mathrm{Si}-\mathrm{O}-\mathrm{Si}$ bond is formed as a water molecule is displaced.

The covalent bond is the main reason for irreversible bonding between the interfaces. The method is robust and can easily sustain high pressure applications. In this study, the oxidising power of piranha solution is utilized to modify the surface property of PDMS to achieve bonding. Although the bonding process has been widely demonstrated, shown to be robust, and proven to be compatible in most of the microfluidic applications, a quantitative study of its bonding effect has never been reported in detail. 
Another issue in using PDMS as a structural material for microfluidic devices is related to its swelling properties when in contact with organic solvents. This obstacle is typically influenced by various factors such as the chemical properties of solvents used, the dimension of the polymer network (i.e., volume and total surface area) [11], as well as the operating temperature of the polymer swelling. This problem rarely attracts attention from researchers although ignoring it may cause relatively important consequences. The use of a large amount of organic solvent throughout the fabrication process, for example the cleaning process, may increase the risk of distortion of the desired dimensions of the microchannels.

In the first part of this paper, a quantitative study on the use of Piranha solution for PDMS-PDMS bonding will be reported. The bonding strength of homogeneous PDMS-PDMS interfaces is tested using four different combinations of piranha solutions with various types of acids, namely concentrated sulfuric acid (99.999 wt\% purity, Sigma-Aldrich), nitric acid (63 wt \%, Sigma-Aldrich), hydrochloric acid (63 wt \%, Sigma-Aldrich) and phosphoric acid ( $85 \mathrm{wt} \%$, Sigma-Aldrich) to hydrogen peroxide (Maersk) respectively in a 3:1 weight ratio. This simple bonding method provides an easy and powerful strategy for irreversible bonding of PDMS-PDMS microfluidic devices with its specific needs and features.

The second part of the paper involves study of PDMS swelling using a weight change method in which PDMS samples were drenched into different organic solvents for durations of up to a week. To the best of our knowledge, this is the first time that the rates of swelling of PDMS under the influence of various solvents has been reported.

In the last section, the effect of Piranha solutions on PDMS surfaces and the influence of the thickness of PDMS on its swelling behavior will be discussed.

\section{Theory}

\subsection{Bonding Mechanism}

The chemical reaction between concentrated sulfuric acid with hydrogen peroxide, generates hydronium ions, bisulphate ions and a reactive atomic oxygen species, as shown in Equation (1) [12]. The formation of a reactive atomic oxygen species is the key leading to the formation of an oxidized PDMS surface because it attacks the $\mathrm{Si}-\mathrm{CH}_{3}$ bonds in PDMS to form a silanol group ( $\mathrm{Si}-\mathrm{OH}$ ), as shown in Figure 1(a-c) [13]. The increase in the concentration of hydroxyl groups leads to the formation of strong intermolecular forces [13]. In addition, the reactive atomic oxygen species also reacts with water simultaneously to form a hydroxyl ion, $\mathrm{OH}^{-}$. The displaced $-\mathrm{CH}_{3}$ entities rapidly react with $\mathrm{H}+$ in the solution to form methane. Thus, when two oxidized PDMS interfaces are brought into conformal contact, the silanol groups merge with each other to form Si-O-Si bonds by displacing a water molecule from the contacting silanol groups. These covalent bonds results in a strong and irreversible seal between the layers. The bonding can withstand up to $210-350 \mathrm{kPa}$ of air pressure, compared to liquid flow which is normally applied at $60 \mathrm{kPa}[14,15]$. 
Figure 1. Methyl group in a Polydimethyl Siloxane (PDMS) network replaced by a Silanol group. (a) Methyl group being attacked by the reactive atomic oxygen species, generated in Piranha Solution. $\mathrm{CH}_{3} \mathrm{O}^{-}$intermediate is formed (b) Unstable $\mathrm{CH}_{3} \mathrm{O}^{-}$is detached from $-\mathrm{Si}-\mathrm{O}-$ branch (c) The vacancy in the structure is taken by $\mathrm{OH}^{-}$to form a silanol group.

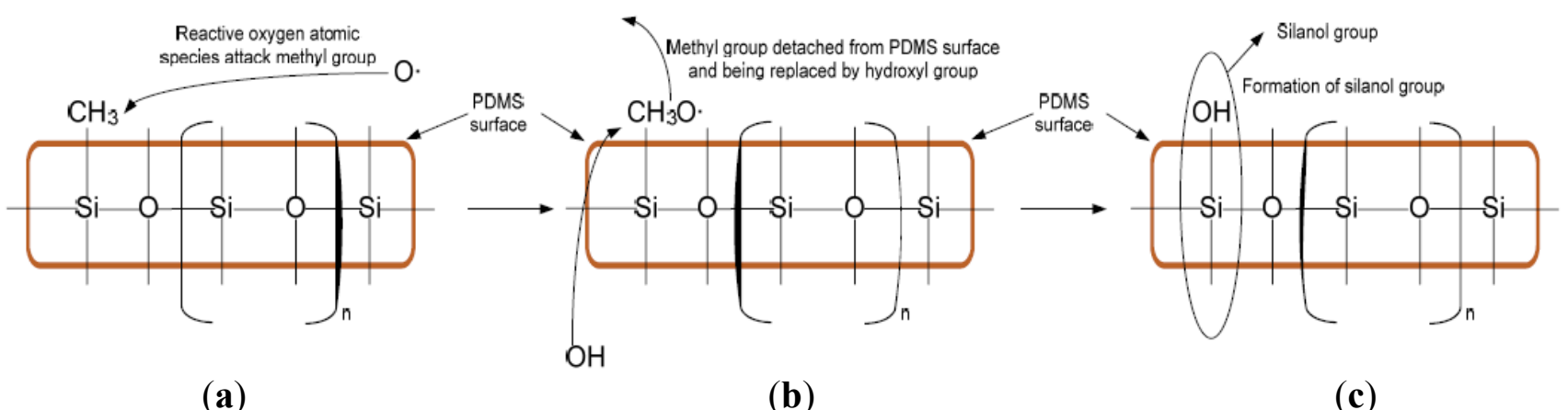

Silanol groups generated by Piranha solution are known to be highly polar in nature, which changes the surface of the PDMS from hydrophobic into hydrophilic [16]. The contact angle of water droplets is expected to be reduced as more silanol groups are formed on the PDMS surface, indicating an increase in hydrophilicity.

$$
\mathrm{H}_{2} \mathrm{SO}_{4}+\mathrm{H}_{2} \mathrm{O}_{2} \rightarrow \mathrm{H}_{3} \mathrm{O}^{+}+\mathrm{H}_{2} \mathrm{O}_{4}^{-}+\mathrm{O}
$$

Equation (1) shows that the concentration of acid and the dissociation strength of the acid are the determining factors in determining the aggressiveness of the piranha solution. A diluted acid mixed with hydrogen peroxide compared to a strong acid may not generate similar effects of attack on the same material.

Since Piranha solutions with concentrated sulfuric acid and hydrogen peroxide pose serious handling and storage issues, replacement with other acids has been investigated to reduce the risk in handling and storage while still achieving the desired bonding strength.

\subsection{Swelling of PDMS}

PDMS is commonly known as a polymer that swells with non-polar solvents (i.e., hydrocarbons, toluene, dichloromethane) due to empty networks in the polymer. Solubility parameters, $\delta\left(\mathrm{cal}^{1 / 2} \mathrm{~cm}^{-3 / 2}\right)$, have been shown to be one of the most convenient ways of correlating swelling [17,18]. An organic solvent has a solubility parameter similar to that of PDMS and can cause a higher degree of swelling. Silanol groups formed at the expense of methyl groups $\left(\mathrm{Si}-\mathrm{CH}_{3}\right)$ on the PDMS surface will condense with appropriate groups $(-\mathrm{OH},-\mathrm{COOH}, \mathrm{C}=\mathrm{O})$ on another surface when they are brought into conformal contact [19-21].

Swelling by organic solvents not only increases the mass of the PDMS device but also causes changes in the dimensions of the microchannels or microstructures in the device, induces distortion, bending, a change in flow characteristics and ultimately leads to a change in flow profile and residence time in the device. This is sometimes important especially when chemical reactions are involved. In addition, swelling of the polymer network also causes delamination of the PDMS-PDMS bonded surfaces [22]. Understanding the swelling behavior of PDMS with various solvents is hence crucial as it serves as a thumbnail in designing PDMS based microfluidic devices. 


\section{Experimental Section}

\subsection{Surface Treatment with Different Piranha Solutions and Tensile Stress Test}

PDMS monomer (Sylgard 184 Silicone Elastomer Kit, Dow Corning) was mixed with its curing agent in the volume ratio of 10:1. The mixture was degassed before it was poured into a mold. Strings were embedded into the PDMS liquid before it was solidified during thermal curing at $65{ }^{\circ} \mathrm{C}$ for $60 \mathrm{~min}$. The samples were then cut into $2 \mathrm{~cm} \times 2 \mathrm{~cm}$ squares, cleaned with methanol (Sigma-Aldrich, 99\%) and dried with nitrogen gas. Piranha solutions were prepared by mixing hydrogen peroxide with concentrated sulfuric acid, nitric acid, hydrochloric acid and phosphorous acids respectively. All of the above solutions were prepared at a weight ratio of hydrogen peroxide to acid of 1:3. Then, the surfaces of the PDMS were drenched with the Piranha solution for times ranging from 15-75 s. The oxidized polymer was then cleaned with DI water, dried with nitrogen gas and kept in conformal contact before a thermal curing process of $70{ }^{\circ} \mathrm{C}$ was applied for $60 \mathrm{~min}$.

Strength measurement of PDMS-PDMS bonding was carried out using a tensile tester (Llyod Instrument, LR50K Plus). It is a 'pull-to-break' strategy in which temporal stress is recorded until the device finally fails. The strength of the strings was initially measured for characterization. During the experiment, strings were first tightened at clamps, then the upper end of the string was pulled upwards at $100 \mathrm{~mm} / \mathrm{min}$ while the lower end of the string remained fixed until the bonded PDMS-PDMS sample detached. A graph of temporal stress applied was plotted after the test. The experiments were repeated five times for each sample as a repeatability and reliability check.

To analyze the quality of samples with excess Piranha solution treatment, the PDMS pieces were examined under a scanning electron microscope (SEM, Quanta400F, FEI)

\subsection{Swelling in PDMS Samples}

A large piece of PDMS was prepared using the method mentioned above. The samples used for swelling were then cut into sizes of $2 \mathrm{~cm}(l) \times 2 \mathrm{~cm}(w) \times 2 \mathrm{~mm}(h)$.

In each experiment, the gross weight of the PDMS samples was recorded before immersion into a solvent. Then, the weight gain of the PDMS samples was recorded every $5 \mathrm{~min}$ for the first $60 \mathrm{~min}$, followed by every $10 \mathrm{~min}$ up to $120 \mathrm{~min}$. Subsequent readings were recorded after $24 \mathrm{~h}$ immersion in the solvents. In each measurement step, the PDMS sample was dried rapidly after removing from the solvent, weighed on a mass balance and re-immersed into the solvent. The weight measurement was completed within $30 \mathrm{~s}$ to minimize the amount of solvent evaporating from the PDMS surface. The amount of swelling with each solvent was then recorded for a week. A total of 18 solvents from different chemical families was utilized in this experiment. The above procedures were repeated at least five times for each sample.

In most cases, the density of the PDMS sample was higher than that of the solvent in which full immersion was viable. In denser solvents such as DMSO and the buffer solutions, fine strands of stainless steel wires were wound around the sample in order to achieve full immersion.

In order to study the influence of swelling on interface bonding, pieces of the PDMS samples with dimensions mentioned previously, drenched in concentrated sulfuric acid-based Piranha solution for $45 \mathrm{~s}$, were bonded into sample thicknesses of 1:1 and 1:2 respectively. The samples were then 
immersed in $25 \mathrm{~mL}$ of hexane for $10 \mathrm{~min}$. The samples were then removed from the solvents for observation.

\subsection{Measurement of Contact Angle}

Pieces of the PDMS samples were drenched into piranha solutions for a duration of time in order to modify the surface properties. The samples were then cleaned thoroughly with cleaning agents and dried with nitrogen gas after removal from the Piranha solution. A contact angle goniometer (Model 250, Rame-Hart) was then used to measure the contact angle of distilled water droplets that were randomly dispersed on the PDMS surface. Five droplet measurements were taken for each piece of PDMS sample.

\section{Result and Discussion}

\subsection{Tensile Strength Measurement}

While the string was being pulled, the tensile stress in the string increased until a point at which the string broke and the tensile stress dropped drastically. The maximum tensile stress before the detachment is referred to as the critical tensile stress. A graph of temporal tensile strength was reported after each experiment of tensile strength measurement, as shown in Figure 2. Initial tests show that the strings can sustain an average critical tensile stress of $46.2 \mathrm{kPa}$ for $9.3 \mathrm{~s}$ before breaking. When two pieces of PDMS without treatment were put together, an average $21 \mathrm{kPa}$ of tensile stress was required to pull them apart.

Figure 2. Graph depicted of the tensile tester when the sample was pulled apart with a speed of $100 \mathrm{~mm} / \mathrm{min}$ for samples drenched in concentrated sulfuric acid for $60 \mathrm{~s}$. The tensile stress in the string increased until the PDMS sample broke away after which the tensile stress dropped significantly.

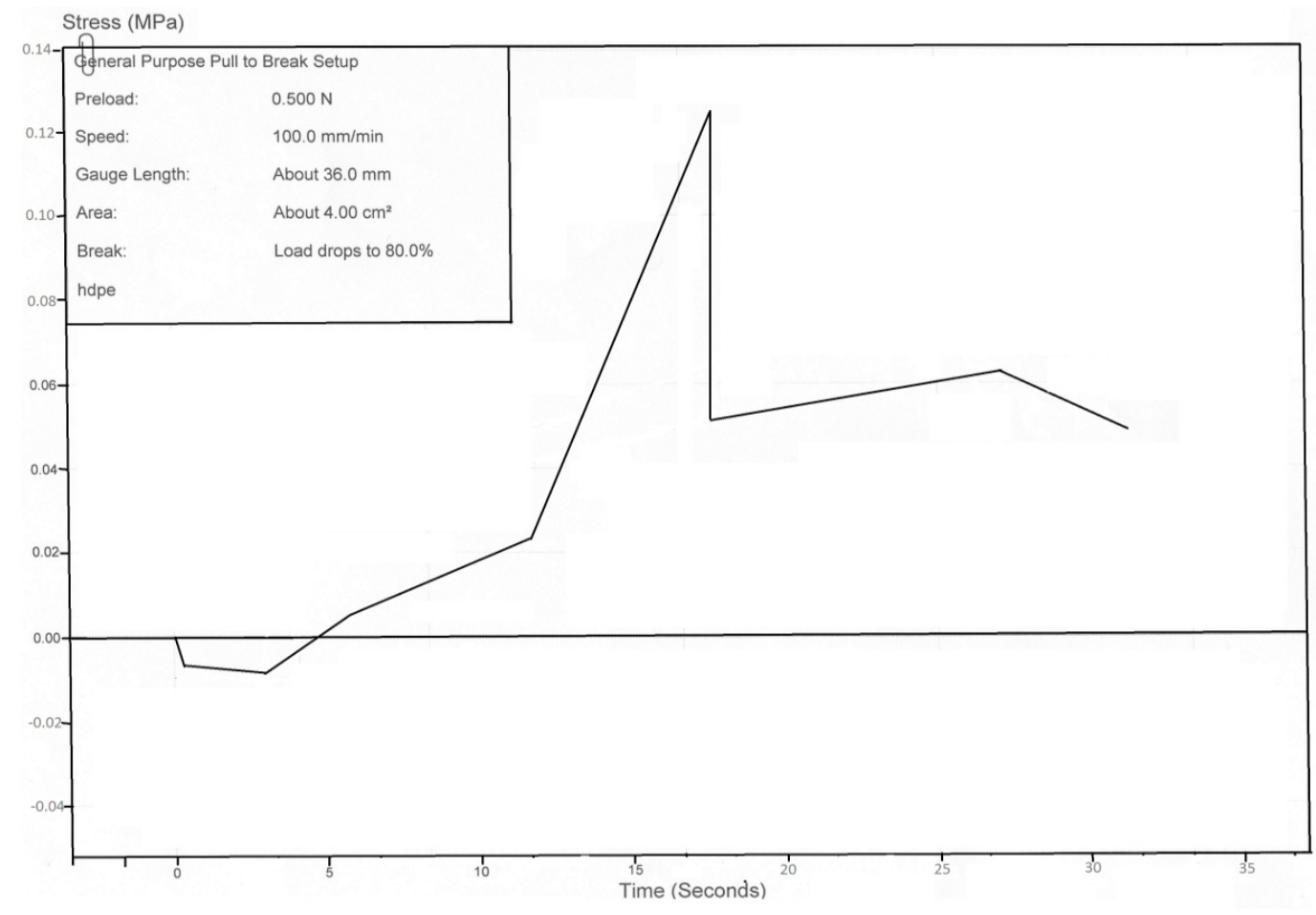


The experiment was also repeated by replacing concentrated sulfuric acid with hydrochloric acid and phosphoric acid in hydrogen peroxide with the same weight ratio, but the bonding failed as the tensile strength was approximately $20 \mathrm{kPa}$, which is similar to the values obtained without treatment as in previous cases.

As shown in Figure 3, the drenching time of the PDMS surface in the oxidizing solution has a significant influence on the critical strength of bonding. Longer drenching times allow oxidation on the PDMS surface to take place and hence generate higher critical tensile stress between the bonded surfaces. Bonding of PDMS-PDMS surfaces using Piranha solution based on concentrated sulfuric acid initially shows an increased critical strength when the drenching time is increased.

Figure 3. Critical Tensile Stress of bonding and its sustainable time against drenching time of PDMS pieces in sulfuric acid and nitric acid-based piranha solution respectively. Solid lines refer to the data of Critical Tensile Strength while the dotted lines refer to sustainable time before string breakup.

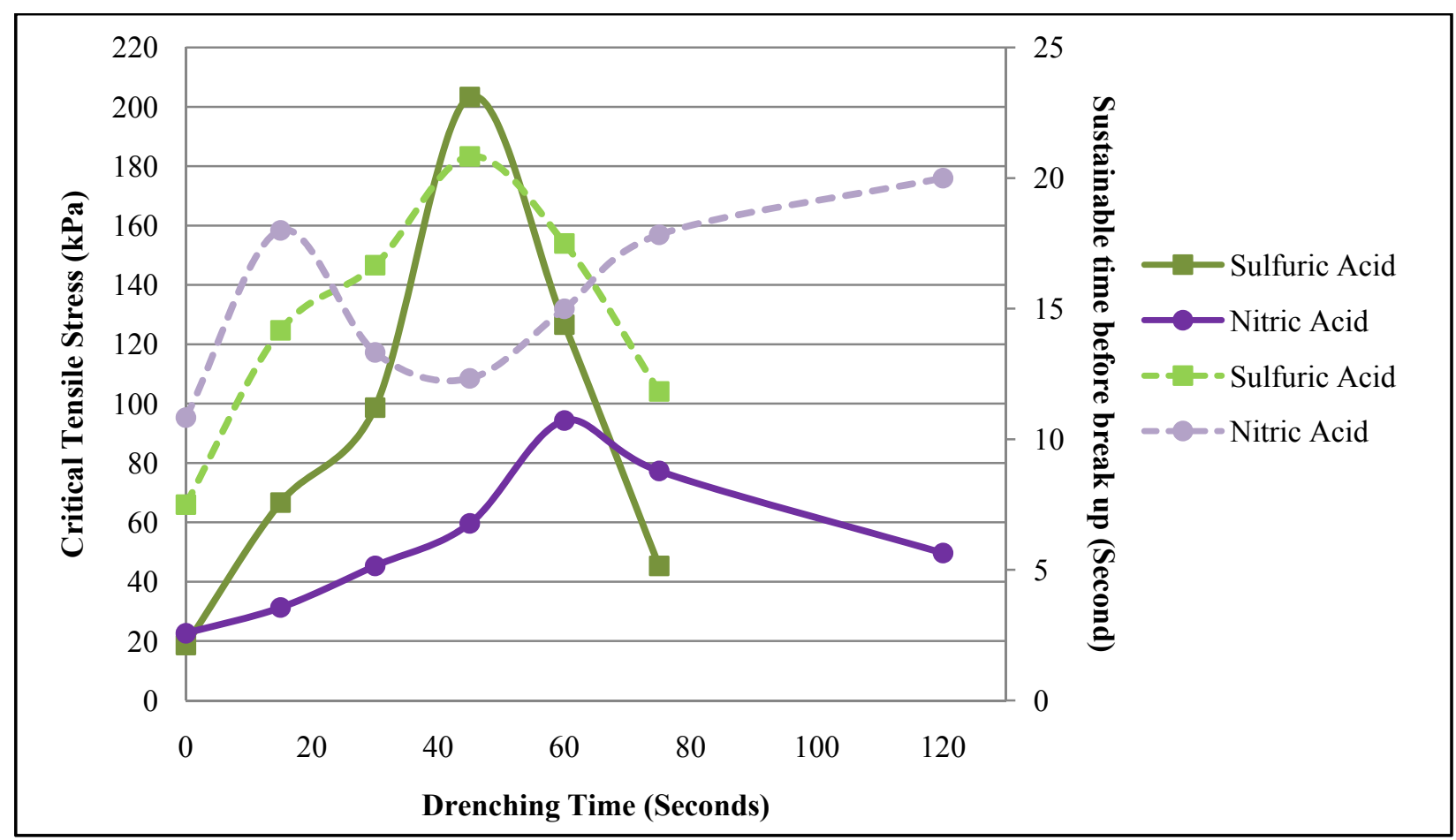

For the case of concentrated sulfuric acid-based Piranha solution, the sustainable duration demonstrates an almost similar trend to the critical tensile strength, as shown in Figure 3, in which the maximum critical tensile strength and maximum sustainable time before breakup is achieved with $45 \mathrm{~s}$ of drenching time. However, there is no predictable pattern for nitric acid-based Piranha solution. As the increase in drenching time gives an increase in the critical tensile stress, the sustainable time peaks at $18 \mathrm{~s}$ and drops to a minimum after a further $12.3 \mathrm{~s}$. The sustainable time before string breakup then increases proportionally with the drenching time. In addition, although critical tensile strengths are reduced after formation of a silica layer on the PDMS surfaces for bonding, the sustainable duration before breakup continues to increase. At $120 \mathrm{~s}$ of drenching time, the sustainable time for nitric acid-based bonding is approximately $20 \mathrm{~s}$, comparable to $20.8 \mathrm{~s}$ for that of concentrated sulfuric acid-based bonding. 
The experiment shows that the number of hydrogen ions in the piranha solution is a determining factor, which depends on the dissociation strength of the acids. Concentrated sulfuric acid is known to have a high dissociation constant and is almost completely dissociated in aqueous solution. It contains more hydrogen ions than nitric acid and hence a shorter time is required to oxidise the surface for bonding. A higher concentration of hydrogen ions is indicative of a higher degree of oxidation and hence results in longer endurance of PDMS-PDMS bonding before detachment. Phosphoric acid and hydrochloric acid, which have low dissociation constants, may not generate sufficient $\mathrm{H}^{+}$for subsequent reactions to fully replace the methyl groups with silanol groups in the PDMS structure.

Prolonged contact between the PDMS surface with piranha solution results in more methyl groups being removed and replaced by $-\mathrm{OH}$ groups in the PDMS network. $\mathrm{H}^{+}$in the solution subsequently freed from silanol groups combines with $\mathrm{CH}_{3}$. free radicals to form methane while a network of $\mathrm{O}-\mathrm{Si}-\mathrm{O}$, or silica, is formed at the surface of the PDMS, resulting in scratches at the surface of the PDMS, as shown in Figure 4. The silica layer prevents the bonding mechanisms taking place when it is in contact with another piece of treated PDMS, thus contributing to the drop in the critical tensile stress. This is particularly noticeable for bonding with sulfuric acid-based piranha solution, in which the critical tensile strength drops significantly from $202 \mathrm{kPa}$ to $45 \mathrm{kPa}$ when the drenching time is extended from $40 \mathrm{~s}$ to $75 \mathrm{~s}$. For sulfuric acid-based piranha solution, a maximum critical tensile strength of $202 \mathrm{kPa}$ is achieved with $40 \mathrm{~s}$ of drenching time while for nitric acid-based Piranha solution, a drenching time of $60 \mathrm{~s}$ only gives a critical tensile strength of $95 \mathrm{kPa}$.

Figure 4. SEM images of PDMS surfaces. (a) Surface of untreated PDMS before immersion into piranha solution; (b) Oxidized surface of PDMS after excessive exposure in piranha solution at 400× magnification; (c) SEM image of cracks as seen in Figure 2(b) at $1,600 \times$ magnification.
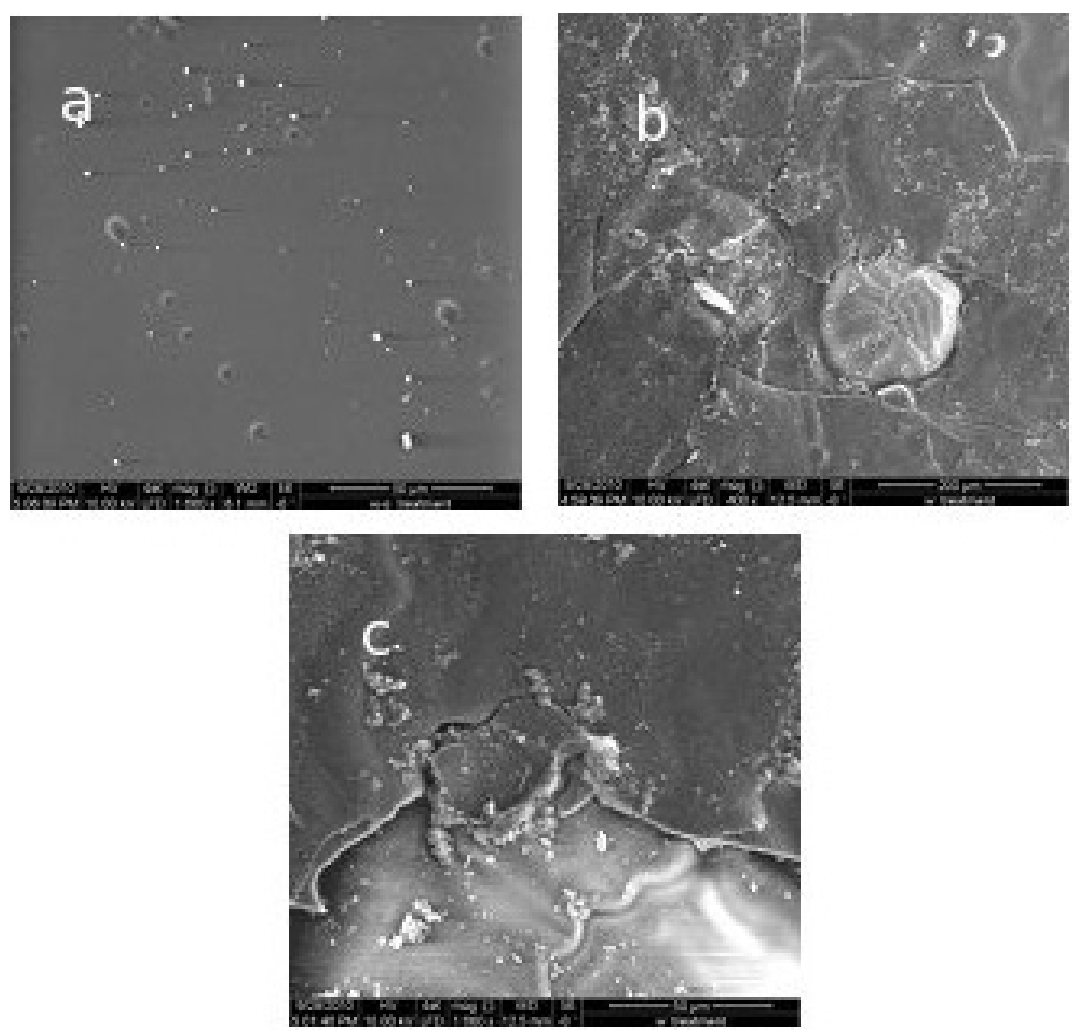


\subsection{PDMS Swelling}

As shown in Figure 5, 5 the most soluble solvents in order of decreasing solubility are hexane, toluene, ethyl acetate, n-propyl alcohol and acetone. However, only hexane, toluene and ethyl acetate cause a large degree of swelling with at least $70 \%$ increase in weight of the PDMS samples throughout the duration of the experiment while the remainder of the solvents cause only a maximum of $20 \%$ increase in weight of the PDMS samples. The behavior of PDMS towards hexane, toluene and ethyl acetate is highly interesting. While PDMS has the highest rate of swelling in the first 15 min in contact with hexane, the rate of swelling decreases gradually until it reaches a steady state in approximately 40 min. Prolonged immersion of the PDMS sample in hexane causes negligible change in its weight. For toluene and ethyl acetate, the solvents have a relatively low rate of swelling compared to hexane, but a steady state is difficult to achieve even when submersion was carried out for $18 \mathrm{~h}$. Overall, only hexane and toluene are able to cause more than $100 \%$ swelling throughout the duration of the experiment. Other solvents do not swell PDMS significantly, achieving a steady state of swelling within $5 \mathrm{~min}$ and a maximum swelling of $7 \%$, as shown in Figure 6 . Based on the swelling trend, the amount of swelling can be compensated for and built in during manufacture of the components if the exposure time of a component to a particular solvent is known.

Figure 5. Rates of PDMS sample swelling, based on percentage of weight increase, when immersed in different solvents for a duration time up to $24 \mathrm{~h}$. Organic solvents which causes less than $10 \%$ of swelling by weight in the figure are reproduced in Figure 6.

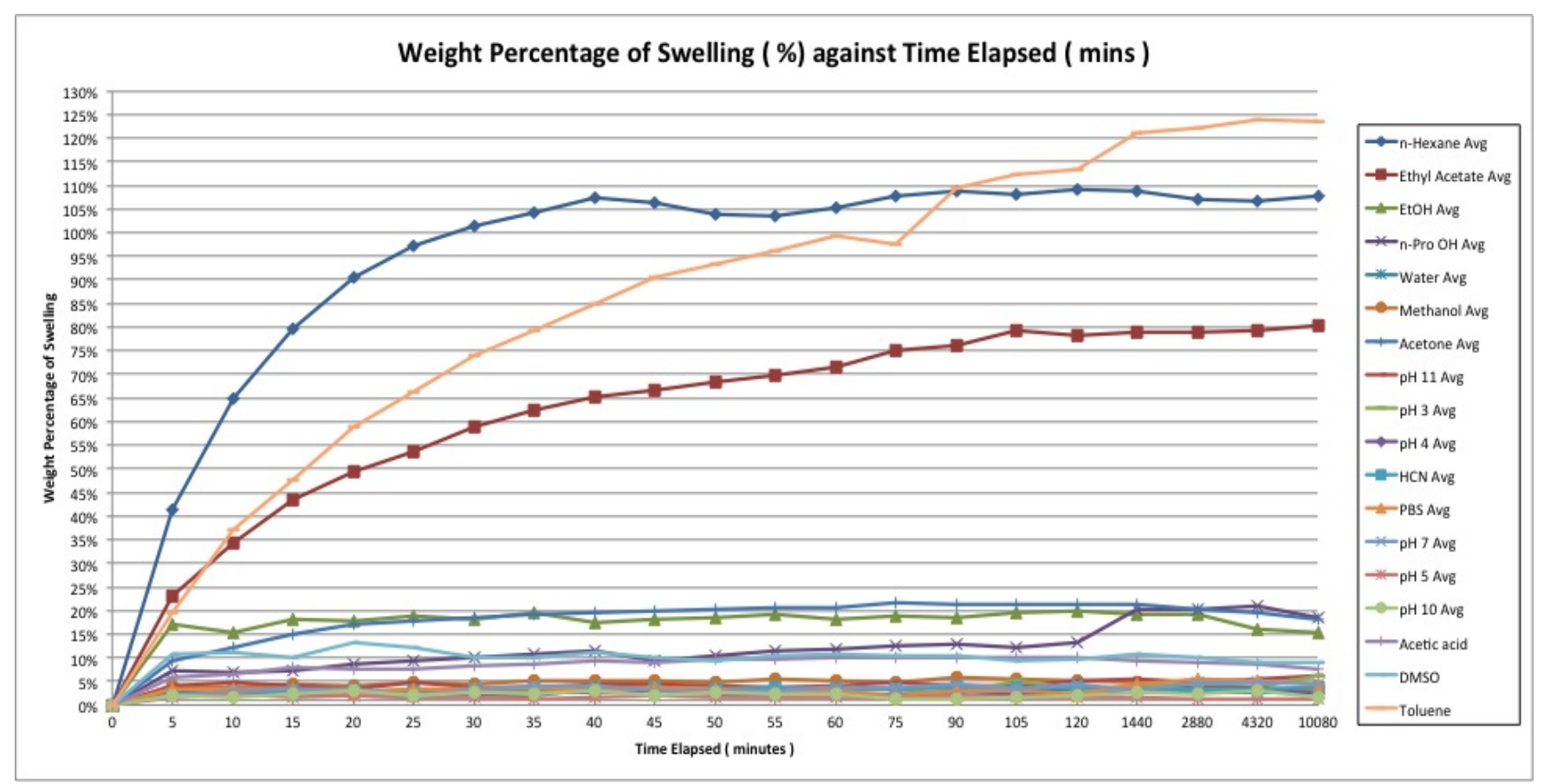


Figure 6. Organic solvents which cause less than 10\% of swelling in PDMS for duration of one week.

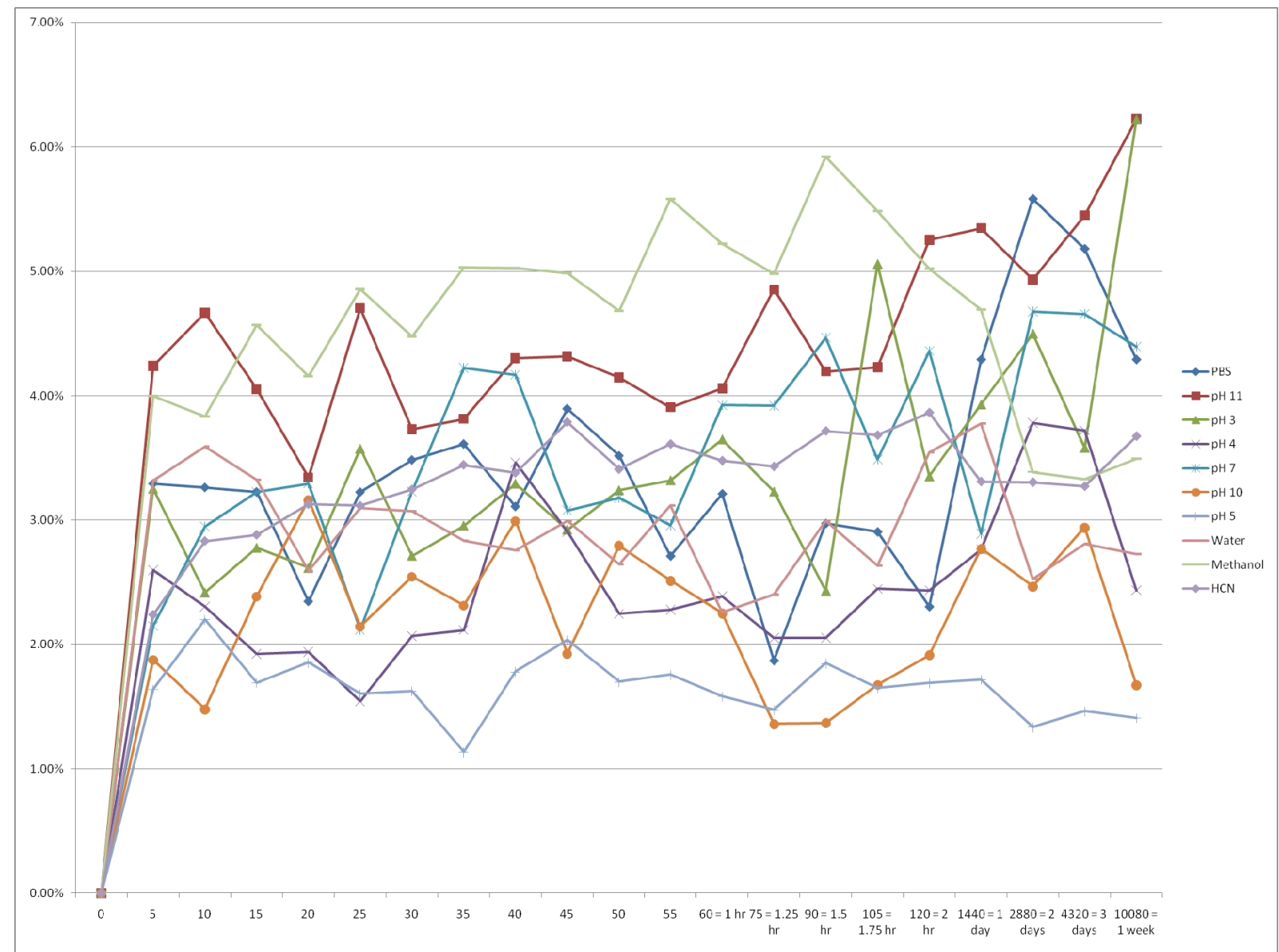

Our experimental data shows close agreement to that reported by Lee et al. [17], as shown in Table 1.

Table 1. Comparison between our experimental results (Swelling of Polydimethyl Siloxane (PDMS) 10 min after submersion in organic solvents.) with that reported by Lee et al. [17]. Both experimental data show similar trends and ranking in swelling properties. The differences in percentage of swelling are due to different measurement methods as well as to sample size and dimension. The size and dimensions of the samples are not drawn to scale.

Our Experimental Result

(In Decreasing Order of Swelling Rate after 10 min $^{1}$ )

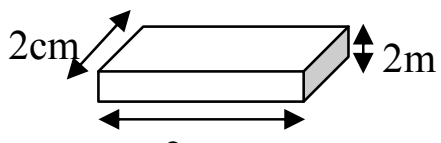

$2 \mathrm{~cm}$

1. n-Hexane (65\%)

2. Ethyl acetate $(34.5 \%)$
Experimental Result from Lee et al. [17]

(In Decreasing Order of Swelling Extent after 24 h)

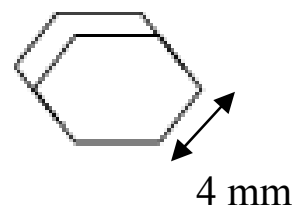

1. n-Hexane (35\%)

2. Ethyl acetate $(18 \%)$ 
Table 1. Cont.

\begin{tabular}{|c|c|}
\hline Our Experimental Results & Experimental Results from Lee et al. [17] \\
\hline 3. Ethanol $(15 \%)$ & 3. n-Propyl alcohol (9\%) \\
\hline 4. Acetone $(12.5 \%)$ & 4. Acetone $(6 \%)$ \\
\hline 5. DMSO $(11.5 \%)$ & 5. Ethyl alcohol (4\%) \\
\hline 6. n-Propyl alcohol (7\%) & No comparison \\
\hline 7. Acetic acid $(6 \%)$ & 6. Methanol (2\%) \\
\hline 8. Buffer solution of $\mathrm{pH} 11^{2}$ & No comparison \\
\hline 9. Water ${ }^{2}$ & 7. Acetonitrile (1\%) \\
\hline 10. Methanol ${ }^{2}$ & No comparison \\
\hline 11. Buffer solution of $\mathrm{pH} 7^{2}$ & No comparison \\
\hline 12. Acetonitrile ${ }^{2}$ & 8. DMSO $(0 \%)$ \\
\hline 13. $\mathrm{PBS}^{2}$ & 9. Water $(0 \%)$ \\
\hline 14. Buffer solution of $\mathrm{pH} 5^{2}$ & No comparison \\
\hline 15. Buffer solution of $\mathrm{pH} 3^{2}$ & No comparison \\
\hline 16. Buffer solution of $\mathrm{pH} 4^{2}$ & No comparison \\
\hline 17. Buffer solution of $\mathrm{pH} 10^{2}$ & No comparison \\
\hline
\end{tabular}

${ }^{1}$ The swelling rate is calculated based on mass change in the first ten min, during which the swelling has been most apparent; ${ }^{2}$ Percentage of swelling less than $5 \%$.

Discerning the swelling rate of PDMS based on the weight method provides a simpler, less costly approach than other methods. The use of dimension changes for example to determine the swelling ratio is far from accurate owing to the fact that evaporation occurs upon contact with air, and the sample starts to shrivel and distort seconds after it is taken out from the solvent, thus prohibiting precise measurement of the dimensions.

In fact, there are three methods to reduce the influence of the solvents on microfluidic devices fabricated from PDMS. One of the methods is to assess the degree of swelling in a pre-study and allow room for swelling to take place, so that at steady state after swelling, the dimensions of the microchannels or microchambers are exactly the desired dimension. Another approach is to limit the contact time between the devices with the solvents so that the degree of swelling is reduced while the third approach is to seek a solvent replacement that can complete its work but have less influence on the PDMS. The following study was completed to identify solvents to reduce contact times.

Figure 7. PDMS-PDMS samples of thickness ratio 1:1(a) and 1:2(b) were prepared by drenching the surfaces in concentrated sulfuric acid-based piranha solution for $45 \mathrm{~s}$; (c,d) the samples were immersed in hexane for $10 \mathrm{~min}$; (e) little distortion with no significant delamination in the sample, (f) drastic delamination leads to failure of the sample.
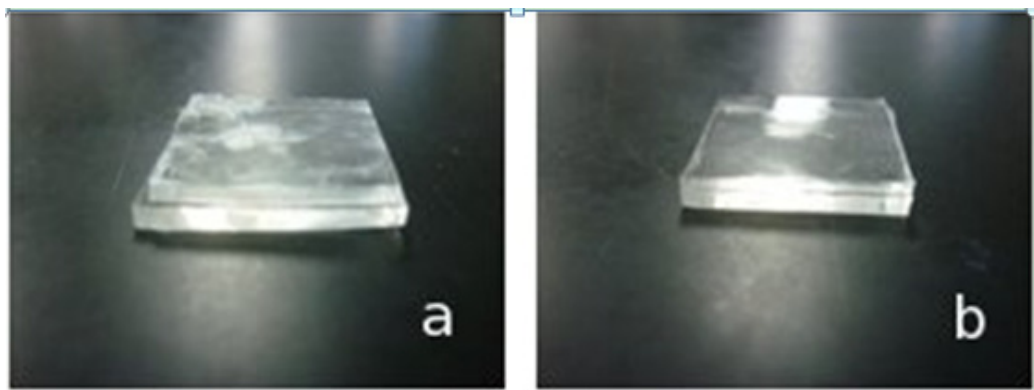
Figure 7. Cont.
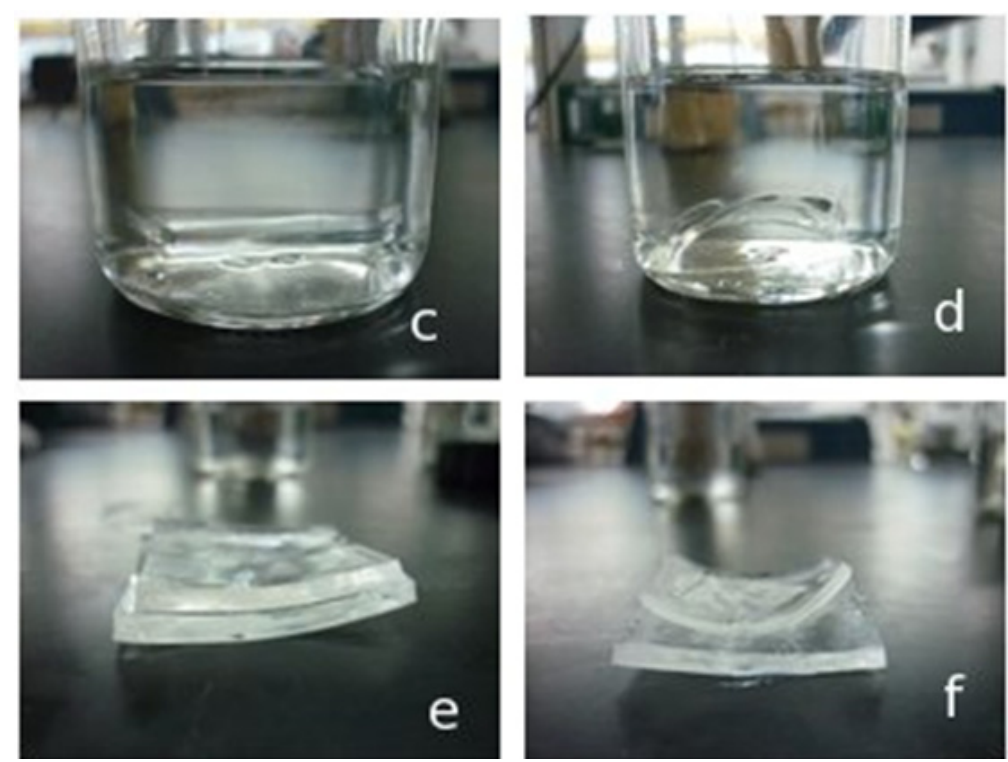

Swelling by organic solvents has a noticeable influence on the bonding strength of PDMS-PDMS interfaces. In Figure 7, after two pieces of PDMS with thickness ratios of 1:1 and 1:2 were immersed in hexane respectively for $10 \mathrm{~min}$, absorption of hexane into the polymer network caused the thinner layer to curve, and the layer to be delaminated. The phenomena are described in Section 4.3 in which samples of ratio $1: 1$ and 1:2 were compared.

\subsection{Effect of Piranha Solutions on PDMS Surfaces}

As shown in Figure 8, treatment of Piranha solutions changes the surface properties of a PDMS surface. Hydrophilicity of the surface increases with prolonged drenching time in piranha solution due to an increase in silanol groups on the PDMS surface until over-treatment in which a silica layer is formed at the surface of the PDMS surface. After surface failure, the contact angles of water on the surface treated with sulfuric acid-based piranha solution show an increase in contact angle with increasing drenching time whereas continuous hydrophilicity is shown on the surface treated with nitric acid-based piranha solution.

Figure 8. Average contact angle of distilled water droplets on a PDMS surface treated with Piranha solution.

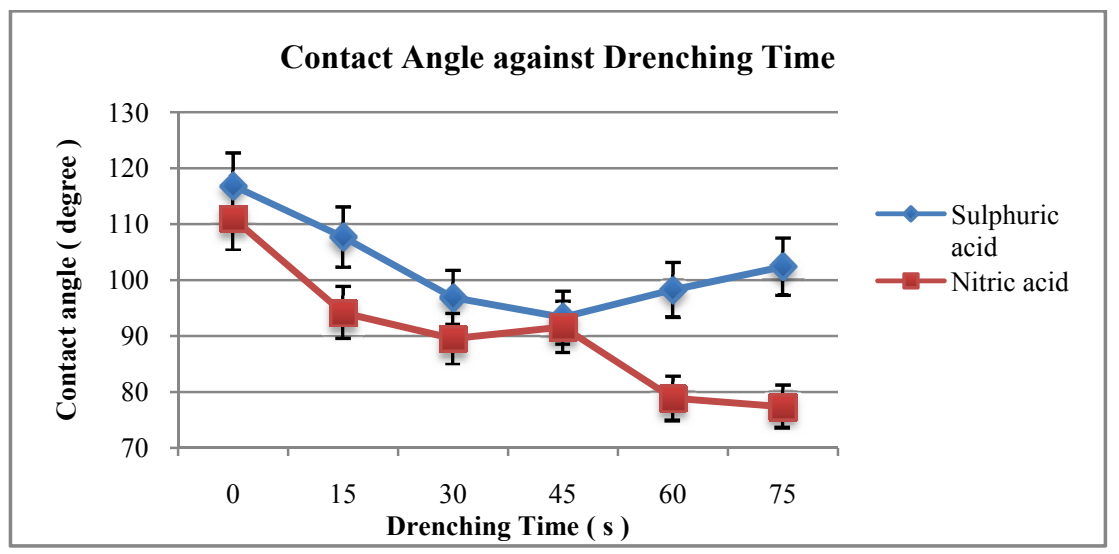




\section{Conclusions}

From the experimental data it can be concluded that the use of sulfuric acid in piranha solution yields samples with the highest bonding strength of $200 \pm 20 \mathrm{kPa}$ as proven by tensile testing whereas a nitric acid-based piranha solution only gives a bonding strength of $150 \pm 20 \mathrm{kPa}$. The exposure time requires critical attention as results indicate a decline in tensile strength after immersion in piranha solution exceeds $45 \mathrm{~s}$. On the other hand, the low dissociation constants of hydrochloric acid and phosphoric acid associated with the low presence of atomic reactive oxygen prohibits the application of these acids in piranha solution.

The experiment on the swelling extent of PDMS immersed in solvents shows that only hexane, toluene and ethyl acetate cause a large degree of swelling in PDMS while the degree of swelling for PDMS samples submerged in other solvents, is relatively small at $20 \%$, with a steady state of swelling achieved within 5 min of submersion.

Swelling is indirectly linked to the solubility parameter and is affected by the molecular weight, and structural similarity of the solvent to the sample. Reducing the area of exposure reduces the absorption rate, and this can be further expanded to study the effect of ratios of height to width upon the degree of distortion of microfluidic channels. PDMS pieces that were bonded using piranha solution and placed into organic solvents produced results illustrating the effect of thickness ratios on bonding strength after swelling. Bonds remained intact after swelling provided the thickness ratios did not differ much.

This study is important for researchers and industry in several ways. First Piranha solution is a low cost yet reliable method for the bonding of PDMS-PDMS microfluidic devices. If the devices are to be used in low pressure flow, nitric acid-based piranha solution is a feasible option as it is cheaper and safer to handle. In addition, care has to be taken when fabricating and bonding microfluidic channels with vast differences in thickness as delamination and distortion may occur which could ruin the entire system.

\section{Acknowledgments}

This work was funded by Fundamental Research Grant Scheme (FRGS/2/2010/TK/UNIM/03/3) under Ministry of Higher Education, Malaysia. Assistance from Dawn Leow and Wai-Siong Chai in carrying out relevant experiments is appreciated.

\section{References}

1. Whitesides, G.M. The origin and the future of microfluidics. Nature 2006, 442, 368-373.

2. Shilpiekandula, V.; Burns, D.J.; El Rifai, K.; Youcef-Toumi, K.; Li, S.; Reading, I.; Soon, F.Y. Metrology of microfluidic devices: A review. ICOMM 2006, 49, 1-6.

3. Neethirajan, S.; Kobayashi, I.; Nakajima, M.; Wu, D.; Nandagopal, S. Microfluidics for food, agriculture and biosystems industries. Lab Chip 2011, 11, 1574-1586.

4. Gorkin, R.; Park, J.; Siegrist, J.; Amasia, M.; Lee, B.S.; Park, J.; Kim, J.; Kim, H.; Madou, M.; Cho, Y. Centrifugal microfluidics for biomedical applications. Lab Chip 2010, 10, 1758-1773. 
5. Duffy, D.C.; Olivier, J.C.M.; Schueller, J.A.; Whitesides, G.M. Rapid prototyping of microfluidic systems in poly(demethylsiloxane). Anal. Chem. 1998, 70, 4974-4984.

6. Duffy, D.C.; Schueller, J.A.; Brittain, S.T.; Whitesides, G.M. Rapid prototyping of microfluidic switches in poly(dimethylsiloxane) and their actuation by electro-osmotic flow. J. Micromech. Microeng. 1999, 9, 211-217.

7. Kim, J.; Chaudhury, M.K. Corona-Discharge-Induced Hydrophobicity Loss and Recovery of Silicones. In Proceedings of Annual Report Conference on Electrical Insulation and Dielectric Phenomena, Austin, TX, USA, 17-20 October 1999; Volume 2, pp. 703-706.

8. Eddings, M.A.; Johnson, M.A.; Gale, B.K. Determining the optimal PDMS-PDMS bonding technique for microfluidic devices. J. Micromech. Microeng. 2008, 18, 067001.

9. Lee, N.Y.; Chung, B.H. Novel poly(dimethyl siloxane) bonding strategy via room temperature "Chemical gluing”. Langmuir 2008, 25, 3861-3866.

10. McDonald, J.C.; Duffy, D.C.; Anderson, J.R.; Chiu, D.T.; Wu, H.K.; Schueller, O.J.A.; Whitesides, G.M. Fabrication of microfluidic systems in poly(dimethylsiloxane). Electrophoresis 2000, 21, 27-40.

11. Dangla, R.; Gallaire, F.; Baround, C.N. Microchannel deformations due to solvent-induced PDMS swelling. Lab Chip 2010, 10, 2972-2978.

12. Damon, Diemente. The hydronium ion: How do we know? J. Chem. Educ. 1991, 68, 568-269.

13. Chabinye, M.L.; Chiu, D.T.; Mcdonald, J.C.; Strook, A.D.; Christian, J.F.; Karger, A.F.; Whitesides, G.M. An integrated fluorescence detection system in poly(dimethylsiloxane) for microfluidic applications. Anal. Chem. 2001, 73, 4491-4498.

14. Mcdonald, J.C.; Duffy, D.C.; Anderson, J.R.; Chiu, D.T.; Wu, H.; Schueller, O.J.A.; Whitesides, G.M. Fabrication of microfluidic systems in polydimethyl siloxane. Electrophoresis 2000, 21, 27-40.

15. Nguyen, N.T.; Werely, S.T. Fundamentals and Applications of Microfluidics; Artech House: Norwood, MA, USA, 2002; pp. 247-292.

16. Hillborg, H.; Gedde, U.W. Hydrophobicity changes in silicone rubbers. IEEE Trans. Dielectr. Electr. Insul. 1999, 5, 701-717.

17. Lee, J.N.; Park, C.; Whitesides, G.M. Solvent compatibility of poly(dimethylsiloxane)-based microfluidic devices. Anal. Chem. 2003, 75, 6544-6554.

18. Chaudhury, M.K.; Whitesides, G.M. Direct measurement of interfacial interaction between semispherical lenses and flat sheets of poly(dimethylsiloxane) and their chemical derivatives. Langmuir 1991, 7, 1013-1025.

19. Chaudhury, M.K.; Whitesides, G.M. Correlation between free energy and surface constitution. Science 1991, 255, 1230-1232.

20. Morra, M.; Occhiello, E.; Marola, R.; Garbassi, F.; Humphrey, P.; Johnson, D. The aging of oxygen plasma-treated polydimethylsiloxane surfaces. J. Colloid Interface Sci. 1990, 137, 11-24.

21. Buckley, D.J.; Berger, M.; Poller, D. The swelling of polymer systems in solvents. I. Method for obtaining complete swelling-time curves. J. Polym. Sci. 1962, 56, 163-174. 
22. Kim, B.Y.; Hong, L.Y.; Chung, Y.M.; Kim, D.P. Solvent resistant PDMS microfluidic devices with hybrid inorganic/organic polymer coatings. Adv. Funct. Mater. 2009, 19, 3796-3803.

(C) 2012 by the authors; licensee MDPI, Basel, Switzerland. This article is an open access article distributed under the terms and conditions of the Creative Commons Attribution license (http://creativecommons.org/licenses/by/3.0/). 